\title{
A joint process model of consensus and longitudinal dynamics
}

\begin{abstract}
Zita Oravecz ${ }^{\mathrm{a}, \dagger}$ and Joachim Vandekerckhove ${ }^{\mathrm{b}}$
This document was compiled on June 17, 2019

The Extended Condorcet Model allows us to explore interindividual consensus concerning culturally held knowledge. At the same time, it enables a process-level description of interindividual differences in the knowledge a person has of the consensus, their willingness to guess in the absence of knowledge, and their bias in guessing. These person-specific characteristics potentially have an influence on one's everyday life experiences. Here, we develop a cognitive latent variable model in which dynamic process parameters from intensive longitudinal daily life data are systematically linked to parameters of the Extended Condorcet Model. We apply this joint model of consensus and longitudinal dynamics to study whether subjective beliefs on what makes people feel loved are linked to daily life experiences of love.
\end{abstract}

Consensus modeling | hierarchical modeling | ecological momentary assessment

Modeling the processes that generate data has the potential to provide a better understanding of human cognition, emotion, and behavior. Process models offer a framework for testing psychological theories by disentangling and quantifying latent processes that are otherwise confounded in observed data. By design, process model parameters correspond to theory-backed concepts such as cultural consensus knowledge (based on Cultural Consensus Theory; Romney \& Batchelder, 1999) or emotion regulation strength (see, e.g., Oravecz, Tuerlinckx, \& Vandekerckhove, 2016), allowing us to perform inference on these concepts directly. Here we develop a model with which we can explore links between cognitive and temporal (dynamical) processes, with all latent parameters made person-specific and estimated simultaneously in a hierarchical Bayesian framework. More specifically, we show how cognitive process model parameters can serve as predictors for emotional processes unfolding over time, and provide a more nuanced understanding of the links between cognition and emotion.

Because of their explanatory potential, cognitive process models have become increasingly popular in cognitive science (Rouder \& Lu, 2005; Smith \& Batchelder, 2010; Vandekerckhove, Tuerlinckx, \& Lee, 2011), where they have allowed researchers to explore individual differences in high-level, abstract features of behavior. More recently, process models have been extended to fully fledged joint-modeling solutions with which modelers can simultaneously account for behavioral data and exogenous data such as clinical evaluations (Vandekerckhove, 2014) or neurophysiological data (Nunez, Gosai, Vandekerckhove, \& Srinivasan, in press; Schubert, Nunez, Hagemann, \& Vandekerckhove, in press; Turner, Forstmann, Love, Palmeri, \& Van Maanen, 2017), or behavioral data across multiple contexts (Guan, Lee, \& Vandekerckhove, 2015). Key in this endeavour is that the model is constructed such that its parameters have useful psychological interpretations. As an example, the popular diffusion model for two-choice response times (Dutilh, Forstmann, Vandekerckhove, \& Wagenmakers, 2013;

\footnotetext{
${ }^{a}$ Pennsylvania State University; ${ }^{\mathrm{b}}$ University of California, Irvine

${ }^{\dagger}$ To whom correspondence should be addressed. E-mail: zita@psu.edu.

The authors declare no conflicts of interest. ZO and JV were supported by grant \#48192 from the John Templeton Foundation. JV was supported by National Science Foundation grant \#1658303. Code and data are available via osf.io/ft5ns.
}

Vandekerckhove, Verheyen, \& Tuerlinckx, 2010) has parameters for a participant's task ability, caution, prior response bias, and nondecision time - all conceptually interesting features of the cognitive decision-making process.

William $\mathrm{H}$. Batchelder was one of the first to emphasize the practical benefits of individual differences in parameters of cognitive models (Batchelder, 2010). Batchelder's pioneering work put us in mind of Lee Cronbach's (1957) call for unification of the correlational and experimental disciplines of psychology. Where Cronbach lamented the schism, and later celebrated the success of aptitudetreatment interaction research (Cronbach, 1975), Batchelder provided the groundwork for the new discipline of cognitive psychometrics - a successful hybrid of psychometrics and mathematical psychology. The first instances of cognitive psychometrics (Batchelder, 1998; Batchelder \& Riefer, 1999) involved multinomial processing tree models-often with a focus on consensus analysis-where individual differences in competencies need to be taken into account when deriving unknown correct answers, or "cultural consensus", on a set of knowledge or belief items. Batchelder, Anders, and Oravecz (2018) summarize the mathematical formulations and implementations of Cultural Consensus Theory (CCT) models these have been applied to various problems including extracting shared information from eyewitness reports (Waubert de Puiseau, Aßfalg, Erdfelder, \& Bernstein, 2012), perceived personality traits in social networks (Agrawal \& Batchelder, 2012), defining psychological concepts (Oravecz, Faust, Levitis, \& Batchelder, 2015), and what makes people feel loved (Heshmati et al., 2019). Weller (2007) provides a summary of various application in the field of cultural anthropology.

In the current paper, we will combine a cultural consensus theory model and a dynamical model into a single joint process model. The first component-the consensus model-is the EXtended Condorcet Model (ECM; Oravecz, Faust, \& Batchelder, 2014). In the ECM the decision-making processes are characterized by theoretically meaningful parameters that can differ among the individuals, such as their awareness of the consensus or "consensus knowledge", their bias towards guessing that an item is true or "acquiescence", and their willingness to guess when they do not know the answer or "guess rate." Most interestingly, the ECM also allows us to derive the shared consensus in a knowledge domain, 
while also accounting for individual differences.

The second component-the dynamical model-is an OrnsteinUhlenbeck (OU) stochastic differential equation model. Dynamical models are often applied to explore the latent processes that generate observed longitudinal data. For example, when we collect data on people's day-to-day experiences in their natural environments (ecological momentary assessment; Shiffman, Stone, \& Hufford, 2008), we can use a dynamical model to learn about intra-individual psychological processes as they unfold over time. This can help us understand the individual as a "unique system of interacting processes" (Molenaar, 2004). A popular approach for capturing dynamics at a system level is differential equation modeling. Such models have been applied to stress regulation (Deboeck \& Bergeman, 2013), smoking cessation (Trail et al., 2013), emotion regulation (Chow, Ram, Boker, Fujita, \& Clore, 2005), cardiovascular changes (Chow, Lu, Sherwood, \& Zhu, 2016), fear-related regulation (Morales et al., 2018) and more. Here we focus on a stochastic differential equation model based on the OrnsteinUhlenbeck process (see, e.g., Kuppens, Oravecz, \& Tuerlinckx, 2010 , for an application on the dynamics of affect). This dynamical process model enables us to capture regularities underlying longitudinal data on everyday life emotions in terms of an attractor point or "baseline", an intra-individual variability around the attractor or "fluctuations", and a speed of return to baseline (i.e., the absence of inertia) or "recovery". Here, too, the process parameters may differ substantially between individuals.

With these two models, and with two different types of data in hand, we can now ask an interesting new question: Are individual differences in cognitive processes and everyday life experiences connected? Studying these links requires access to both modes of data, a substantively meaningful breakdown of the processes that could generate these data, and a nuanced latent variable model with which we can estimate diverse process model parameters and their relationships simultaneously.

While it would be possible to first acquire parameter estimates from both process models separately and put these into a regression model as predictors and outcomes, such a two-step procedure incurs a risk of introducing bias into the regression coefficient estimations by not taking into account the (joint) uncertainty in the predictors and outcomes (Pagan, 1984) - this risk of biased estimation is one of the main motivations of the joint modeling framework (Vandekerckhove, 2014). Our proposed model includes simultaneous estimation of all model parameters and coefficients, and is implemented in the hierarchical Bayesian framework (Gelman \& Hill, 2007).

Next we introduce the ECM and show how the ECM cognitive process model parameters can enter directly as predictors for the process model parameters of the OU process. Then we continue by showing an application of the framework to study whether beliefs on what makes people feel loved are linked to daily life experiences of love.

\section{The Extended Condorcet Model}

Exploring shared agreement (i.e., consensus) requires $N$ persons responding (e.g., in terms of True, False, or Don't know) to $M$ items from a chosen knowledge domain. By fitting the ECM we can derive the consensus regarding these items in a way that also accounts for individual differences in the decision-making process. The answer from a single person $i=1, \ldots, N$, for item $k=1, \ldots, M$ is denoted by $A_{i k}$, while the consensus answer, item $k$ : "Most people feel loved when someone cares for them"

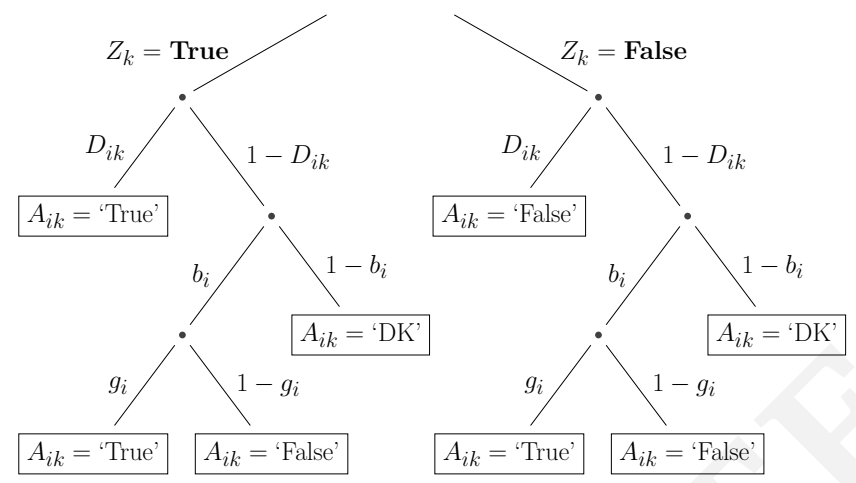

Fig. 1. Decision tree representation of the Extended Condorcet model. Read from top to bottom, the decision tree depicts the steps of the decision process. Presented with item $k$, decider $i$ traverses the tree, choosing each branch with its associated transition probability, until a decision is arrived at.

which is a model parameter, is denoted by $Z_{k}$ for an item $k$. The probability that respondent $i$ knows the consensus answer for item $k$ has probability $D_{i k}$. The probability that respondent $i$ chooses to guess when uncertain about the item is their guess rate, $b_{i}$. If the respondent is not willing to take a guess, it is assumed that they will mark the 'Don't know' ('DK') option, with probability $1-b_{i}$. When it comes to guessing, a respondent has a tendency to mark 'True' with probability $g_{i}$, which captures their acquiescence bias. Figure 1 graphically displays the decision-making processes assumed by the ECM.

By following along the branches of the decision tree in Figure 1 we may calculate the probabilities for each response category. These result in:

$$
\begin{aligned}
& P\left(A_{i k}=\text { 'True' }\right)= Z_{k} \times\left[D_{i k}+\left(1-D_{i k}\right) \times b_{i} \times g_{i}\right] \\
&+\left(1-Z_{k}\right) \times\left(1-D_{i k}\right) \times b_{i} \times g_{i} \\
& P\left(A_{i k}=\text { 'False' }\right)=\quad Z_{k} \times\left(1-D_{i k}\right) \times b_{i} \times\left(1-g_{i}\right)+\left(1-Z_{k}\right) \\
& \times\left[D_{i k}+\left(1-D_{i k}\right) \times b_{i} \times\left(1-g_{i}\right)\right] \\
& P\left(A_{i k}=\text { 'DK' }\right)=Z_{k} \times\left(1-D_{i k}\right) \times\left(1-b_{i}\right) \\
&+\left(1-Z_{k}\right) \times\left[\left(1-D_{i k}\right) \times\left(1-b_{i}\right)\right] .
\end{aligned}
$$

If we now collect the left hand sides into a $3 \times 1$ probability vector

$$
\boldsymbol{P}_{i k}=\left[P\left(A_{i k}=\text { 'True' }\right), P\left(A_{i k}=\text { 'False' }\right), P\left(A_{i k}=\text { 'DK' }\right)\right],
$$

then the responses $A_{i k}$ are assumed to follow a categorical distribution (denoted by Cat):

$$
A_{i k} \sim \operatorname{Cat}\left(\boldsymbol{P}_{i k}\right)
$$

The probability $D_{i k}$ of respondent $i$ answering item $k$ correctly (i.e., according to the consensus) can be made a function of the respondent's consensus knowledge (i.e., ability) and the item's difficulty level, as in a Rasch model (De Boeck \& Wilson, 2004; Rasch, 1960), in order to allow items to differ in difficulty:

$$
\operatorname{logit}\left(D_{i k}\right)=\log \left(\frac{D_{i k}}{1-D_{i k}}\right)=\theta_{i}-\delta_{k}
$$

where $\theta_{i}$ now quantifies $i$ 's consensus knowledge and $\delta_{k}$ is $k$ 's difficulty. The population distribution of $\delta_{k}$ is defined to have mean $0: \delta_{k} \sim N\left(0, \sigma_{\delta}^{2}\right)$. The population distribution for $\theta_{i}$ is defined to 
be normal with mean and variance estimated: $\theta_{i} \sim N\left(\mu_{\theta}, \sigma_{\theta}^{2}\right)$. The other two person-specific parameters, guess rate (willingness to guess; $b_{i}$ ) and acquiescence bias (propensity of guessing true; $g_{i}$ ), are given normal population distributions on the logit scale, for example logit $\left(b_{i}\right) \sim N\left(\mu_{b}, \sigma_{b}^{2}\right)$. The hyperparameters were given uninformative priors: normal distributions with mean 0 and variance 100 for the population means and uniform distributions between 0.001 and 100 on the population standard deviations. The prior on the latent answer key was set to a Bernoulli assigning equal probability to True or False responses: $Z_{k} \sim \operatorname{Bern}(.5)$.

To summarize, the Extended Condorcet Model captures individual differences in the decision-making process by three person-specific parameters: consensus knowledge $\left(\theta_{i}\right)$, guess rate $\left(b_{i}\right)$, and acquiescence $\left(g_{i}\right)$. These will serve as predictors into a process model that captures changes in emotional experiences over time, described next.

\section{Cognitive latent variables in the Ornstein-Uhlenbeck process model}

In EMA studies everyday-life temporal processes can be explored with intensive longitudinal data (ILD). ILD sets are typically made up of relatively many (i.e., more than 50 ) repeated measures over time from more than a handful of people. We will denote the ILD by $Y_{i, s}$, collected at time points $t_{i, 1}, t_{i, 2}, \ldots, t_{i, s}, \ldots, t_{i, n_{i}}$, where index $s$ stands for the $s^{\text {th }}$ measurement occasion of participant $i=$ $1, \ldots, N$. The measurements are typically taken semi-randomly at different times for different participants in the study and the total number of time points $n_{i}$ can also vary over participants.

For every person's data, we can use the Ornstein-Uhlenbeck model's parameters-baseline, recovery, and fluctuations-to disentangle the underlying process that has generated them. It is a continuous-time model that can be used to account for changes from one observation given the other, for example via the conditional distribution of the process (Oravecz, Wood, \& Ram, 2018):

$$
\begin{aligned}
Y_{i, s} \mid Y_{i, s-1} & \sim N\left(m_{i, s}, \sigma_{i, s}^{2}\right) \\
m_{i, s} & =\mu_{i}+e^{-\beta_{i}\left(t_{i, s}-t_{i, s-1}\right)}\left(Y_{i, s-1}-\mu_{i}\right) \\
\sigma_{i, s}^{2} & =\gamma_{i}\left(1-e^{-2 \beta_{i}\left(t_{i, s}-t_{i, s-1}\right)}\right)
\end{aligned}
$$

In this formulation, parameter $\mu_{i}$ is the person-specific baseline, also known as the attractor or attraction point. Variation around this baseline is modeled through $\gamma_{i}$, a person-specific intra-individual variance parameter we will call the fluctuations. The model assumes that there is always some level of attraction, or recovery over time towards the baseline level, and the dynamics of this is modeled through the person-specific $\beta_{i}$ parameter. These three process parameters are depicted in Figure 2. Finally, the first observation, $Y_{i, 1}$, is assumed to come from a normal distribution with the baseline as a mean and the intra-individual variance parameters as variance: $Y_{i, 1} \sim N\left(\mu_{i}, \gamma_{i}\right)$. Next we expound upon the parameterizations, define level-2 distributions and cognitive predictors.

By hierarchically extending the OU model, these three personspecific parameters can be functionally linked to other parameters. By combining the OU model with the ECM into an ECM-OU model, all of the cognitive parameters enter jointly in a single modeling step, ensuring that uncertainty is appropriately propagated from the latent parameter estimates to the regression coefficient estimates. The level-2 distributions (i.e., distributions on the "population" level)

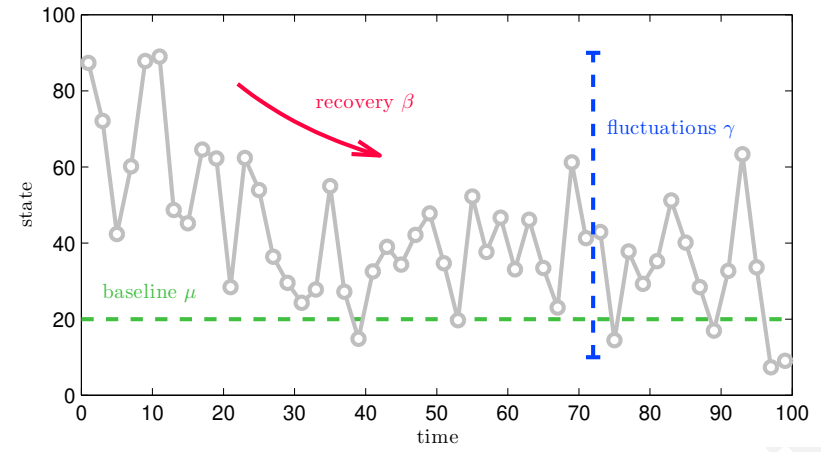

Fig. 2. A cartoon of the Ornstein-Uhlenbeck diffusion process. The jagged pattern shows the stochastic nature of the process. The horizontal dashed line indicates the baseline to which the process tends to return, and the curved arrow indicates the direction of the recovery. The dashed vertical interval illustrates the intra-individual variability or fluctuations.

of the three parameters in the combined model are now the following:

$$
\begin{aligned}
\theta_{i} & \sim N\left(\mu_{\theta}, \sigma_{\theta}^{2}\right), \\
f\left(g_{i}\right) & \sim N\left(\mu_{g}, \sigma_{g}^{2}\right), \\
f\left(b_{i}\right) & \sim N\left(\mu_{b}, \sigma_{b}^{2}\right), \\
\mu_{i} & \sim N\left(\alpha_{\mu}+\alpha_{\theta \mu} \theta_{i}+\alpha_{g \mu} f\left(g_{i}\right)+\alpha_{b \mu} f\left(b_{i}\right), \sigma_{\mu}^{2}\right), \\
\log \left(\gamma_{i}\right) & \sim N\left(\alpha_{\gamma}+\alpha_{\theta \gamma} \theta_{i}+\alpha_{g \gamma} f\left(g_{i}\right)+\alpha_{b \gamma} f\left(b_{i}\right), \sigma_{\gamma}^{2}\right), \\
\log \left(\beta_{i}\right) & \sim N\left(\alpha_{\beta}+\alpha_{\theta \beta} \theta_{i}+\alpha_{g \beta} f\left(g_{i}\right)+\alpha_{b \beta} f\left(b_{i}\right), \sigma_{\beta}^{2}\right) .
\end{aligned}
$$

In the equations above, $\alpha_{\mu}, \alpha_{\gamma}$ and $\alpha_{\beta}$ are intercepts and all three dynamical parameters are regressed on the three cognitive personspecific parameters $\left(\theta_{i}\right.$ : consensus knowledge; $g_{i}$ : acquiescence bias; $b_{i}$ : guess rate; $f(\cdot)$ : logit transformation), with corresponding $\alpha$-s capturing the association strengths. Note that a single model now ties together the two modes of data. The set of model assumptions (4) simultaneously governs the individual participant's EMA data $Y_{i, s}$ through the latent parameters $\left(\mu_{i}, \gamma_{i}, \beta_{i}\right)$ and process equation (3), and their consensus behavior $A_{i, k}$ via Equation 1.

Note that the set of model assumptions above can be restated as a latent variable model with the following structural equation:

$$
\left(\begin{array}{c}
\tilde{\boldsymbol{\theta}} \\
f(\tilde{\boldsymbol{g}}) \\
f(\tilde{\boldsymbol{b}}) \\
\tilde{\boldsymbol{\mu}} \\
\ln (\tilde{\boldsymbol{\gamma}}) \\
\ln (\tilde{\boldsymbol{\beta}})
\end{array}\right)=\left(\begin{array}{ccc}
1 & 0 & 0 \\
0 & 1 & 0 \\
0 & 0 & 1 \\
\alpha_{\theta \mu} & \alpha_{g \mu} & \alpha_{b \mu} \\
\alpha_{\theta \gamma} & \alpha_{g \gamma} & \alpha_{b \gamma} \\
\alpha_{\theta \beta} & \alpha_{g \beta} & \alpha_{b \beta}
\end{array}\right) \times \boldsymbol{\Phi}+\left(\begin{array}{c}
\mu_{\theta} \\
\mu_{g} \\
\mu_{b} \\
\alpha_{\mu} \\
\alpha_{\gamma} \\
\alpha_{\beta}
\end{array}\right) \times \mathbf{1}_{(1 \times N)}
$$

and an error term with a diagonal covariance matrix. Here, all the latent factors are captured in a $3 \times N$ factor matrix $\boldsymbol{\Phi}$. The hybrid process model is thus a cognitive latent variable model (Vandekerckhove, 2014).

Throughout, we use appropriate link functions to map parameters from a limited domain to the full real line. This allows us to place linear regression models on the transformed parameters while simultaneously respecting the parameter's natural domain (e.g., while $b$ and $g$ naturally live on probability scales, we use standard logit functions $f(\cdot)$ to map them to the domain that belongs to the normal distribution).

Priors were again set as uninformative normal distributions, centered at 0 with variance of 100 on the regression coefficients 
$(\alpha-s)$, and uniform distributions between 0.001 and 100 on the population standard deviations $(\sigma-\mathrm{s})$.

\section{Application: Do beliefs on what makes people feel loved predict feeling loved in everyday life?}

Study settings. Fifty-two participants (17 male and 35 female, mean age $=30$ years, $S D=10, \min =19, \max =48$ ) were recruited to participate in an EMA study on daily emotional experiences. During an introductory session participants completed a battery of demographic items and psychological tests, including the evaluation of 60 everyday life scenarios in which people could potentially feel loved ('Felt love scenarios'; see Heshmati et al., 2019). Starting the next day, participants started to receive text messages to complete short web-based surveys via their smart phones. They received these surveys six times daily, on a semi-random schedule, for four weeks. Specifically, the participants' usual waking hours were divided into six equal-length intervals and in each interval a message arrived at a random time, with no two prompts less than 30 minutes apart. Over the course of the four weeks, participants received and responded to up to 168 text-message prompted web-based surveys. Each survey contained approximately 10-12 questions (including items related to their felt level of accomplishment, engagement etc.), but only data coming from the felt love intensity question is analyzed here. Compliance was high, with participants completing an average of $157(S D=15)$ surveys. Participants were paid proportional to their response rate, with a maximum payment of $\$ 200$. The study was overseen by the Penn State University's Institutional Review Board (STUDY1017).

Data. We fit the the ECM-OU model to both modes of data-the consensus data and the longitudinal data-simultaneously. For the ECM, we used respondents' True/False/Don't know responses on the Love scenarios questionnaire. The OU parameters in turn were derived from participants' self-reports about the degree to which they felt loved, based on their response to the question "How much do you feel loved right now?". Participants responded to this question with a visual digital sliding scale, with the two extremes labeled as "Not at all" and "Extremely". Responses were mapped to integers between 0 and 100 respectively. To illustrate these data, Figure 3 shows the responses from four participants over the course of the study. Individual differences in terms of baseline levels (e.g., between Participant 1 and 2) and fluctuations (e.g., between Participant 1 and 3) are apparent. Moreover, we can also see that Participant 1 returns more quickly to their baseline than Participant 4 , for whom the changes in the felt love levels show more inertia.

Implementation. The ECM-OU model was fit to the data in JAGS (Plummer, 2003) and R (R Core Team, 2017), using rjags (Plummer, 2016), and redundantly using JAGS and MATLAB using trinity (Matzke, Boehm, \& Vandekerckhove, 2018). All scripts and data are available via osf.io/ft5ns. We ran 4 MCMC chains with 25,000 iterations each (after 10,000 adaptation steps and an additional 5,000 burnin samples). Convergence was assessed by confirming the $\hat{R}$ value being below 1.1 (Gelman et al., 2013) and by visual assessment of selected trace plots - no problems with convergence were identified.

Results. Individual differences in ECM parameters (consensus knowledge on the scenarios in which people feel loved, guess rate,
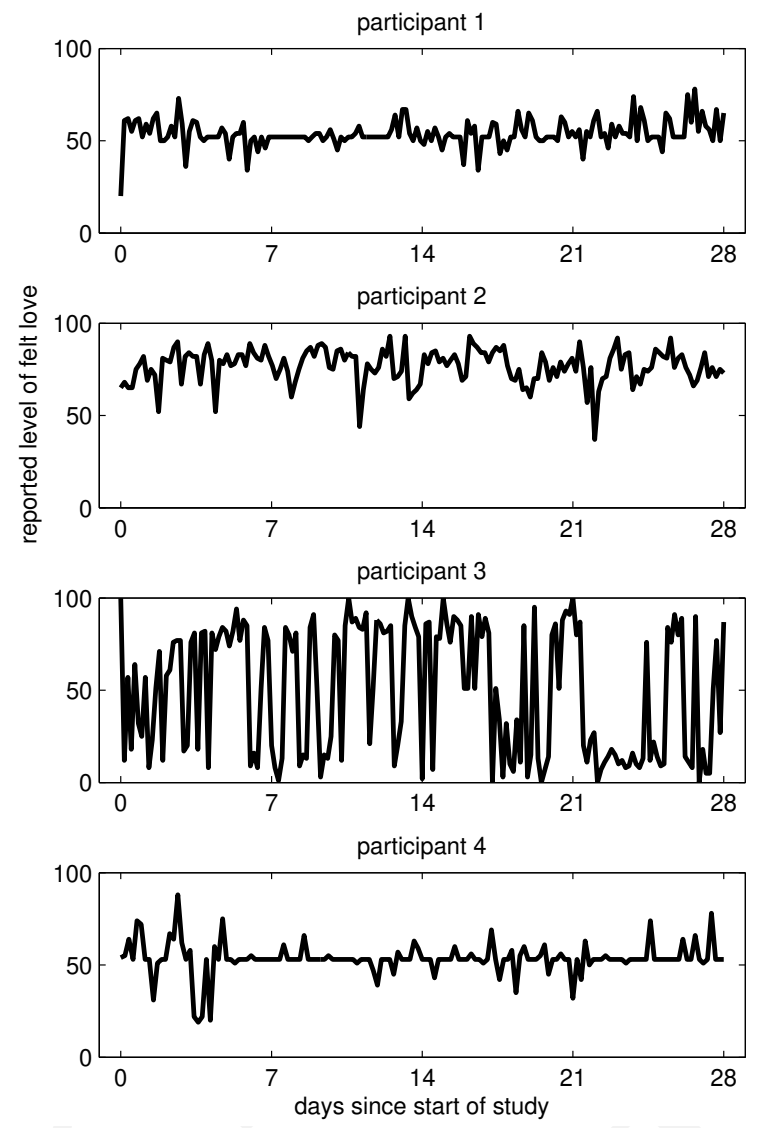

Fig. 3. Self-reported levels of felt love from 4 participants over the course of the study, reported on a scale from 0 (Not at all) to 100 (Extremely). Participant 1 has a lower baseline than Participant 2, less fluctuations than Participant 3 , and quicker return to baseline than Participant 4.

acquiescence bias) and OU-based dynamical parameters (baseline felt love intensity, recovery to the baseline, and fluctuations in felt love) were estimated and connected as described in the set of model assumptions in (4). Table 1 shows the estimated regression coefficients linking the cognitive predictors to the dynamic characteristics, in terms of their posterior point estimates (the posterior mean; third column).

To judge whether a coefficient is meaningfully different from 0 , we delineated a region of practical equivalence (ROPE) around 0 , with limits set to -0.01 and 0.01 . Columns 4 through 6 show the posterior probability of a parameter being below, inside, or above (respectively) this ROPE interval. As a rule of thumb, when $95 \%$ of posterior mass (.95 probability) is on either side of the ROPE, we conclude that the effect is credibly different from 0 . Based on this criterion, we found three credible links: higher consensus knowledge was associated with less fluctuations around the baseline, higher propensity of guessing true was related to faster recovery to the baseline (i.e., less inertia in the experiences), and people who were more willing to guess if they were uncertain tended to have higher baselines.

Figure 4 shows the inferred relationships between the two trios of parameters. In that figure, all parameters have been transformed back to their original scale to reverse the link functions used in the model. That is, while the set of model assumptions in (4) involves only linear equations, these linear functions were applied 


\begin{tabular}{llcccc}
\hline \hline $\begin{array}{l}\text { Cognitive } \\
\text { predictors }\end{array}$ & $\begin{array}{l}\text { Dynamic } \\
\text { parameters }\end{array}$ & $\begin{array}{c}\text { Posterior } \\
\text { mean }\end{array}$ & \multicolumn{3}{c}{ Mass relative to ROPE: } \\
below & inside & above \\
\hline Consensus knowledge & Baseline & -0.60 & .60 & .00 & .40 \\
Consensus knowledge & Recovery & 3.90 & .17 & .00 & .83 \\
Consensus knowledge & Fluctuations & -0.30 & .96 & .01 & .03 \\
Acquiescence bias & Baseline & 0.67 & .25 & .01 & .75 \\
Acquiescence bias & Recovery & 3.98 & .03 & .00 & .97 \\
Acquiescence bias & Fluctuations & 0.01 & .38 & .12 & .50 \\
Guess rate & Baseline & 1.79 & .05 & .00 & .95 \\
Guess rate & Recovery & 1.86 & .28 & .01 & .71 \\
Guess rate & Fluctuations & -0.03 & .60 & .10 & .30 \\
\hline
\end{tabular}

Table 1. Results on regression coefficients linking cognitive predictors to daily life loving experience dynamics. ROPE indicates a region of practical equivalence to 0 and was set between to -0.01 and 0.01 .

to transformed parameters $\log (\gamma), \log (\beta), \operatorname{logit}(g)$ and logit $(b)$. The figure instead shows $\gamma, \beta, g$, and $b$. This results in some highly nonlinear link functions. For example, while the regression in the top left panel of Figure 4 is a standard linear model, the regression in the central panel is a log-logit linear regression. The advantage of this back-transformed plotting is that it yields more readily interpretable scatter plots. The three credible associations highlighted in Table 1 are depicted in red in the figure.

These three credible links suggest an interplay between cognitive beliefs on love and day-to-day experiences of love. Specifically, participants who were more knowledgeable of the consensus on which situations might elicit loving feelings experienced less fluctuations in their everyday intensities of felt love (i.e., they showed generally less variable, more stable profiles). At the same time, participants who were more likely to believe that a potentially loveinducing scenario was indeed love-inducing when they were unsure showed less inertia in felt love intensities; that is, upon being steered away from their baseline they reestablished homeostasis more rapidly. Finally, being willing to guess when uncertain about a scenario appeared to be linked to more intense love experiences in everyday life.

To conclude, imagine a person who knows well the cultural consensus on which daily life scenarios people would feel loved. If this person is uncertain in some cases, they might optimistically presume love is being conveyed. Based on the current findings, such a person would feel loved more intensely than average in daily life, would vary less in terms of the intensity of the experiences and whenever something puts them out of balance they would return to their baseline more quickly.

\section{Discussion}

We successfully formulated a complex joint process model to simultaneously account for cognitive processes, longitudinal dynamics and their interplay. Two modes of data were used to estimate the underlying latent process parameters.

The model was applied in a study of everyday life aspects of love, and meaningful links emerged in terms of how cognitive beliefs connect to everyday life experiences. Interestingly, it was not the participants' level of consensus knowledge that was linked to more intense loving feelings in everyday life, but how much they were willing to guess when they were unsure about a scenario. Knowledge level, however, was meaningfully related to less intraindividual variation, suggesting more stability in love experiences.

Results of this study should be considered within the following limitations. Our conclusions are based on a convenience sample of staff and international students who were staying on campus for

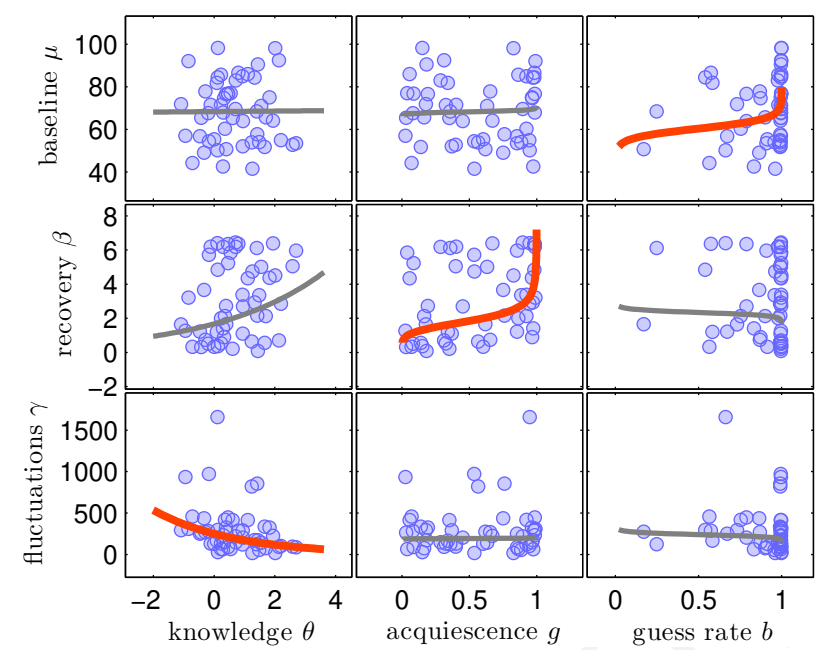

Fig. 4. Pairwise scatter plots and regression lines for the two sets of model parameters within the joint model. While most associations are around zero, three of the scatter plots (from bottom left to top right) show consistent relationships. The regressions are nonlinear because all parameters were transformed back to their natural scales after the linear regression weights were estimated.

the summer break. Moreover, while we found meaningful associations between cognitive characteristics and emotion dynamics, we cannot conclude directionality: it is possible that everyday life experiences shape cognition or vice versa.

\section{References}

Agrawal, K., \& Batchelder, W. H. (2012). Cultural consensus theory: Aggregating signed graphs under a balance constraint. In G. A. M. Shanchieh J. Y. \& M. Endsley (Eds.), Social computing, behavioral-cultural modeling, \& prediction (SBP 2012) (pp. 53-60). Heidelberg: Springer.

Batchelder, W. H. (1998). Multinomial processing tree models and psychological assessment. Psychological Assessment, 10(4), 331.

Batchelder, W. H. (2010). Cognitive psychometrics: Using multinomial processing tree models as measurement tools. In S. Embretson (Ed.), Measuring psychological constructs: Advances in model-based approaches (pp. 71-93). Washington, DC: American Psychological Association Books.

Batchelder, W. H., Anders, R., \& Oravecz, Z. (2018). Cultural consensus theory. In J. Wixted \& E. J. E. Wagenmakers (Eds.), The stevens' handbook of experimental psychology and cognitive neuroscience, volume $v$ : Methodology (pp. 221-264). New York, NY: John Wiley \& Sons.

Batchelder, W. H., \& Riefer, D. M. (1999). Theoretical and empirical review of multinomial process tree modeling. Psychonomic Bulletin \& Review, 6(1), 57-86.

Chow, S.-M., Lu, Z., Sherwood, A., \& Zhu, H. (2016). Fitting nonlinear ordinary differential equation models with random effects and unknown initial conditions using the Stochastic Approximation Expectation Maximization (SAEM) algorithm. Psychometrika, 81, 102-134.

Chow, S.-M., Ram, N., Boker, S. M., Fujita, F., \& Clore, G. (2005). Emotion as thermostat: Representing emotion regulation using a damped oscillator model. Emotion, 5(2), 208-225.

Cronbach, L. J. (1957). The two disciplines of scientific psychology. American psychologist, 12(11), 671. 
Cronbach, L. J. (1975). Beyond the two disciplines of scientific psychology. American psychologist, 30(2), 116.

De Boeck, P., \& Wilson, M. (2004). Explanatory item response models: A generalized linear and nonlinear approach. New York: Springer.

Deboeck, P. R., \& Bergeman, C. S. (2013). The reservoir model: A differential equation model of psychological regulation. Psychological Methods, 18, 237-256.

Dutilh, G., Forstmann, B. U., Vandekerckhove, J., \& Wagenmakers, E.-J. (2013). A diffusion model account of age differences in posterror slowing. Psychology and Aging, 28(1), 64.

Gelman, A., Carlin, J. B., Stern, H. S., Dunson, D. B., Vehtari, A., \& Rubin, D. B. (2013). Bayesian data analysis, Third edition. Boca Raton (FL): Chapman \& Hall/CRC.

Gelman, A., \& Hill, J. (2007). Data analysis using regression and multilevel/hierarchical models. Cambridge: Cambridge University Press.

Guan, M., Lee, M. D., \& Vandekerckhove, J. (2015). A hierarchical cognitive threshold model of human decision making on different length optimal stopping problems. In R. Dale et al. (Eds.), Proceedings of the 37th annual conference of the cognitive science society (pp. 824-829). Austin, TX: Cognitive Science Society.

Heshmati, S., Oravecz, Z., Pressman, S., Batchelder, W., Muth, C., \& Vandekerckhove, J. (2019). What does it mean to feel "loved"? cultural agreement and individual differences. Journal of Social and Personal Relationships, 36(1), 214243.

Kuppens, P., Oravecz, Z., \& Tuerlinckx, F. (2010). Feelings change: Accounting for individual differences in the temporal dynamics of affect. Journal of Personality and Social Psychology, 99, 1042-1060.

Matzke, D., Boehm, U., \& Vandekerckhove, J. (2018). Bayesian inference in psychology, part iii: Bayesian parameter estimation in nonstandard models. Psychonomic Bulletin \& Review, 25, 77-101.

Molenaar, P. C. M. (2004). A manifesto on psychology as idiographic science: Bringing the person back into scientific psychology, this time forever. Measurement: Interdisciplinary Research and Perspectives, 2(4), 201-218.

Morales, S., Ram, N., Buss, K. A., Cole, P. M., Helm, J. L., \& Chow, S.-M. (2018). Age-related changes in the dynamics of fear-related regulation in early childhood. Developmental Science, 21(5), e12633.

Nunez, M. D., Gosai, A., Vandekerckhove, J., \& Srinivasan, R. (in press). The latency of a visual evoked potential tracks the onset of decision making. Neurolmage.

Oravecz, Z., Faust, K., \& Batchelder, W. H. (2014). An extended cultural consensus theory model to account for cognitive processes in decision making in social surveys. Sociological Methodology, 44, 185-228.

Oravecz, Z., Faust, K., Levitis, D., \& Batchelder, W. H. (2015). Studying the existence and attributes of consensus on psychological concepts by a cognitive psychometric model. American Journal of Psychology, 128, 61-75.

Oravecz, Z., Tuerlinckx, F., \& Vandekerckhove, J. (2016). Bayesian data analysis with the bivariate hierarchical OrnsteinUhlenbeck process model. Multivariate Behavioral Research, 51, 106-119.

Oravecz, Z., Wood, J., \& Ram, N. (2018). Fitting continuous time stochastic process models in the Bayesian framework. In van Montfort, O. J, \& V. E. Manuel (Eds.), Continuous time modeling in the behavioral and related sciences (pp. 55-78). Berlin: Springer.

Pagan, A. (1984). Econometric issues in the analysis of regressions with generated regressors. International Economic Review, 25, 221-247.

Plummer, M. (2003). JAGS: A program for analysis of Bayesian graphical models using Gibbs sampling. In Proceedings of the 3rd international workshop on distributed statistical computing (DSC 2003) (pp. 20-22).

Plummer, M. (2016). rjags: Bayesian graphical models using mcmc. (R package version 4-6)

R Core Team. (2017). R: A language and environment for statistical computing. Vienna, Austria.

Rasch, G. (1960). Probabilistic models for some intelligent and attainment tests. Copenhagen, Denmark: Danish Institute for Educational Research.

Romney, A. K., \& Batchelder, W. H. (1999). Cultural consensus theory. Cambridge, MA.: The MIT Press.

Rouder, J., \& Lu, J. (2005). An introduction to Bayesian hierarchical models with an application in the theory of signal detection. Psychonomic Bulletin \& Review, 12, 573-604.

Schubert, A.-L., Nunez, M. D., Hagemann, D., \& Vandekerckhove, J. (in press). Individual differences in cortical processing speed predict cognitive abilities: A model-based cognitive neuroscience account. Computational Brain \& Behavior.

Shiffman, S., Stone, A. A., \& Hufford, M. R. (2008). Ecological momentary assessment. Annual Review of Clinical Psychology, 4(1), 1-32. (PMID: 18509902)

Smith, J. B., \& Batchelder, W. H. (2010). Beta-mpt: Multinomial processing tree models for addressing individual differences. Journal of Mathematical Psychology, 54(1), 167 - 183. (Contributions of Mathematical Psychology to Clinical Science and Assessment)

Trail, J. B., Collins, L. M., Rivera, D. E., Li, R., Piper, M. E., \& Baker, T. B. (2013). Functional data analysis for dynamical system identification of behavioral processes. Psychological Methods, 19, 175-182.

Turner, B. M., Forstmann, B. U., Love, B. C., Palmeri, T. J., \& Van Maanen, L. (2017). Approaches to analysis in modelbased cognitive neuroscience. Journal of Mathematical Psychology, 76, 65-79.

Vandekerckhove, J. (2014). A cognitive latent variable model for the simultaneous analysis of behavioral and personality data. Journal of Mathematical Psychology, 60, 58-71.

Vandekerckhove, J., Tuerlinckx, F., \& Lee, M. D. (2011). Hierarchical diffusion models for two-choice response times. Psychological Methods, 16, 44-62.

Vandekerckhove, J., Verheyen, S., \& Tuerlinckx, F. (2010). A crossed random effects diffusion model for speeded semantic categorization decisions. Acta Psychologica, 133(3), 269-282.

Waubert de Puiseau, B., Aßfalg, A., Erdfelder, E., \& Bernstein, D. M. (2012). Extracting the truth from conflicting eyewitness reports: A formal modeling approach. Journal of Experimental Psychology: Applied, 18, 390-403.

Weller, S. C. (2007). Cultural consensus theory: Applications and frequently asked questions. Field Methods, 19, 339-368. 